\section{(6) OPEN ACCESS}

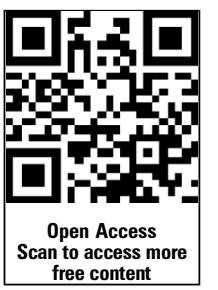

- Additional material is published online only. To view please visit the journal online (http://dx.doi.org/10.1136/ jnnp-2012-303816)

${ }^{1}$ Clinical Research Unit, School of Health and Social Care, Bournemouth University, Bournemouth, Dorset, UK ${ }^{2}$ Person Centred Research Centre, School of Rehabilitation and Occupation Studies, AUT University, Auckland, New Zealand

${ }^{3}$ MS Research Unit, Bristol and Avon MS Clinical Centre, Frenchay Hospital, Bristol UK ${ }^{4}$ Health Economics Group, University of Exeter Medical School, University of Exeter, Exeter, UK

${ }^{5}$ Dorset MS Service, Poole Hospital NHS Foundation Trust, Poole, UK

${ }^{6}$ Faculty of Health and Social Care, Hull University, Hull, UK

\section{Correspondence to} Dr Sarah Thomas, Clinical Research Unit, School of Health and Social Care, Bournemouth University, Bournemouth, Dorset BH1 3LT UK;

saraht@bournemouth.ac.uk

Received 2 August 2012 Revised 8 March 2013 Accepted 14 April 2013 Published Online First 22 May 2013

\footnotetext{
To cite: Thomas $S$, Thomas PW, Kersten $\mathrm{P}$ et al. I Neurol Neurosurg Psychiatry 2013;84: 1092-1099.
}

\title{
A pragmatic parallel arm multi-centre randomised controlled trial to assess the effectiveness and cost-effectiveness of a group-based fatigue management programme (FACETS) for people with multiple sclerosis
}

\author{
Sarah Thomas, ${ }^{1}$ Peter W Thomas, ${ }^{1}$ Paula Kersten, ${ }^{2}$ Rosemary Jones, ${ }^{3}$ Colin Green, ${ }^{4}$ \\ Alison Nock, ${ }^{5}$ Vicky Slingsby, ${ }^{5}$ Angela Davies Smith, ${ }^{3}$ Roger Baker, ${ }^{1}$ \\ Kathleen T Galvin, ${ }^{6}$ Charles Hillier ${ }^{5}$
}

\section{ABSTRACT}

Background Fatigue is a common and troubling symptom for people with multiple sclerosis (MS).

Aim To evaluate the effectiveness and cost-effectiveness of a six-session group-based programme for managing MS-fatigue (Fatigue: Applying Cognitive behavioural and Energy effectiveness Techniques to lifeStyle (FACETS)).

Methods Three-centre parallel arm randomised controlled trial with economic evaluation. Patients with MS and significant fatigue were randomised to FACETS plus current local practice (FACETS) or current local practice alone (CLP), using concealed computergenerated randomisation. Participant blinding was not possible. Primary outcomes were fatigue severity (Fatigue Assessment Instrument), self-efficacy (Multiple SclerosisFatigue Self-Efficacy) and disease-specific quality of life (Multiple Sclerosis Impact Scale (MSIS-29)) at 1 and 4 months postintervention (follow-up 1 and 2). Quality adjusted life years (QALYs) were calculated (EuroQoL 5Dimensions questionnaire and the Short-form 6-

Dimensions questionnaire).

Results Between May 2008 and November 2009, 164 patients were randomised; primary outcome data were available for 146 (89\%). Statistically significant differences favour the intervention group on fatigue selfefficacy at follow-up 1 (mean difference (MD) 9, 95\% Cl (4 to 14), standardised effect size (SES) $0.54, p=0.001$ ) and follow-up 2 (MD 6, 95\% Cl (0 to 12), SES 0.36, $\mathrm{p}=0.05)$ and fatigue severity at follow-up $2(\mathrm{MD}-0.36$, $95 \% \mathrm{Cl}(-0.63$ to -0.08$)$, SES $-0.35, \mathrm{p}=0.01)$ but no differences for MSIS-29 or QALYs. No adverse events reported. Estimated cost per person for FACETS is $£ 453$; findings suggest an incremental cost-effectiveness ratio of $f 2157$ per additional person with a clinically significant improvement in fatigue.

Conclusions FACETS is effective in reducing fatigue severity and increasing fatigue self-efficacy. However, it is difficult to assess the additional cost in terms of costeffectiveness (ie, cost per QALY) as improvements in fatigue are not reflected in the QALY outcomes, with no significant differences between FACETS and CLP. The strengths of this trial are its pragmatic nature and high external validity.

Trial registration: Current Controlled Trials ISRCTN76517470.

\section{INTRODUCTION}

Fatigue is one of the most commonly reported and disabling symptoms of multiple sclerosis (MS); ${ }^{1}$ $65 \%$ of people with MS consider it one of their three most troubling symptoms. ${ }^{2}$ MS-fatigue differs from tiredness experienced by healthy people in both severity and impact. It can have a profound impact on individuals' lives; limiting or preventing participation in everyday activities, work, leisure and social pursuits; and reducing psychological well-being. ${ }^{3}$ It is one of the key precipitants of early retirement ${ }^{4}$ and its 'invisible' nature can make it difficult for others to understand. ${ }^{5}$ The pathophysiology of fatigue is unclear but likely to be multi-factorial. ${ }^{6}$

In North America treatments such as energy effectiveness approaches have been shown to be moderately helpful. ${ }^{7}$ A systematic review of the treatment of fatigue in MS highlighted a lack of high quality studies, particularly those evaluating psychological and psychosocial approaches. ${ }^{9}$ Although the important relationships between physical and psychological aspects of MS-fatigue are recognised, ${ }^{10}$ to date there has been just one trial that has used a cognitive behavioural approach to managing fatigue in people with MS. ${ }^{11}$ The intervention consisted of one-to-one clinical psychologist-delivered cognitive behavioural therapy and was shown to be effective in reducing fatigue. However, in the UK National Health Service (NHS) and elsewhere, psychologists working with people with MS are scarce, and thus this approach may prove impractical. ${ }^{12}$

We developed a group-based manualised intervention for the management of MS-fatigue (Fatigue: Applying Cognitive behavioural and Energy effectiveness Techniques to lifeStyle (FACETS))..$^{13}$ The FACETS intervention uses health professionals routinely involved in managing MS, supported by a clinical psychologist. This pragmatic two parallel arm multi-centre randomised controlled trial (RCT) is a formal evaluation of the effectiveness and cost-effectiveness of FACETS when added to current local practice. ${ }^{14}$ 


\section{METHODS}

The trial was overseen by a steering committee and is reported in accordance with the Consolidated Standards of Reporting Trials guidelines for non-pharmacological trials. ${ }^{15}$ Ethical approval was obtained from the South West-Central Bristol Research Ethics Committee (ref: 08/H0106/2). All participants gave written informed consent before taking part. The trial sponsor was Poole Hospital NHS Foundation Trust.

The main inclusion criteria were: (1) clinically definite MS diagnosis, (2) fatigue impacting on daily life (Fatigue Severity Scale total score $>4)^{16}$ and (3) ambulatory. The main exclusion criteria were: (1) having taken part in a fatigue programme in the last year, (2) cognitive impairments, (3) a relapse in the previous 3 months or (4) having started treatment with disease modifying or antidepressant drugs within the previous 3 months. The full eligibility criteria are described in the protocol. ${ }^{14}$ Participants were recruited in three UK centres (Poole, Bristol, Southampton/Portsmouth) from primary or secondary care, or via MS Society newsletters/websites. Recruitment took place from May 2008 to November 2009.

\section{Intervention (FACETS)}

The manualised group-based FACETS programme is described elsewhere ${ }^{13}$ and is based upon a conceptual framework integrating elements from cognitive behavioural, social-cognitive, energy effectiveness, self-management and self-efficacy theories. The aim of the intervention is to help people normalise their fatigue experiences, learn helpful ways of thinking about fatigue and use available energy more effectively. The intervention consists of six sessions ( $~ 90$ min duration) held weekly and facilitated in groups of 6-12 by two health professionals with experience of working with people with MS and group-work (such as occupational therapists, nurses or physiotherapists). The intervention is highly structured. Each session follows the same general format, namely, facilitator-delivered presentations, flipchart discussions, group activities and homework. The facilitator manual provides guidance on preparation and delivery, detailed session content, notes and suggested timings, and a checklist of facilitator objectives as well as signposts to additional resources. Sessions are delivered via PowerPoint; hence can be easily replicated. A companion participant handbook, along with existing information booklets, reinforces programme content. The incorporation of flipchart exercises, group discussions and homework activities enables the tailoring of content to the individual circumstances and goals/priorities of group members. Participants receiving the intervention continued to have access to services available as part of their usual local care.

FACETS was delivered in hotel meeting-room facilities, with the exception of one centre, where it was held in a rehabilitation hospital. Apart from one MS specialist nurse, facilitators were either occupational therapists or physiotherapists. Facilitators were all qualified to a senior/advanced level (in the UK minimum of a Band 7 on the NHS Agenda for Change (AfC) grading structure). ${ }^{17}$ Facilitators were trained to deliver the intervention at 1-day workshops and psychological advice and debriefing were available for facilitators throughout the trial.

Prior to this full scale trial, we carried out a small pilot study $(n=18)$ delivering the group-based intervention once in each of the three centres (Poole, Bristol, Southampton) and collecting preoutcome and postoutcome measurements. This enabled us to test trial procedures, check clarity of and adherence to the manual and explore transferability of the intervention.

\section{Current local practice}

Participants randomised to the CLP arm of the trial received current local practice only. This could have ranged from general advice and information provision about MS-fatigue to more detailed individualised management advice from a variety of health professionals. Inevitably, there will have been variations in the exact composition of what was usually provided, within and between centres, depending on local resources and patient need. Collecting detailed information at an individual level on the type and quantity of advice received as part of current local practice was not deemed feasible. However, we consider this variation to be a strength of the trial as it increases its applicability to a wider range of centres. Individuals who had attended a fatigue management programme in the past year were excluded from the trial as were those who had received a fatigue intervention from a health professional in the past 3 months (see protocol). ${ }^{14}$ To increase external validity, no attempt was made in the FACETS arm to restrict or control participants' access to current local practice or to standardise it across healthcare settings or treatment arms. When we refer to the FACETS arm, participants in this arm also received current local practice.

To ensure good allocation concealment, random allocation was email based and administered by the statistician who was masked to the identity of participants. Randomisation used a computerised random number generator. Once a block of up to 24 participants from a centre had provided informed consent, they were formally entered onto the trial database and an anonymised list of their identification numbers was sent to the statistician who randomly allocated half to the FACETS arm and half to the CLP arm.

\section{Masking}

The nature of the trial meant that participant blinding was not possible. All outcomes were self-reported using validated measures.

\section{Outcome measures}

Demographic information collected is reported in table 1.

For those allocated to the FACETS arm, outcomes were measured 1 week (baseline) before the start of FACETS and 1 month (follow-up 1) and 4 months (follow-up 2) after the final session. Participants assigned to the CLP arm completed all outcome measures within an identical time frame to those in the FACETS arm (see published protocol ${ }^{14}$ and results tables). All questionnaire outcomes were self-reported for both groups, presented in a booklet format with a large font and administered and returned through the post. This meant that participants could pace the completion of the outcome measures in their own homes.

\section{Primary outcome measures}

These were: (1) fatigue severity (Global Fatigue Severity (GFS) subscale of the Fatigue Assessment Instrument (FAI)), ${ }^{18}$ (2) disease-specific quality of life (QOL) (total score on the Multiple Sclerosis Impact Scale, V.1 (MSIS-29, V.1)) ${ }^{19}$ and (3) self-efficacy for managing fatigue (Multiple Sclerosis-Fatigue Self-Efficacy scale-adapted from the Control subscale of the MS Self-Efficacy scale). ${ }^{20}$

\section{Secondary outcome measures}

See protocol ${ }^{14}$ and online web supplement for further detail. These included: (1) subscales of the FAI, ${ }^{18}$ (2) the Fatigue Symptom Inventory, ${ }^{21}$ (3) the Hospital Anxiety and Depression 
Table 1 Descriptive statistics for demographic and baseline characteristics of participants

\begin{tabular}{|c|c|c|}
\hline & $\begin{array}{l}\text { FACETS } \\
(\mathrm{n}=84)\end{array}$ & $\begin{array}{l}\text { CLP } \\
(n=80)\end{array}$ \\
\hline \multicolumn{3}{|l|}{ Gender (n (\%)) } \\
\hline Female & $61(73 \%)$ & $58(73 \%)$ \\
\hline Male & $23(27 \%)$ & $22(28 \%)$ \\
\hline \multicolumn{3}{|l|}{ Age (years) } \\
\hline Mean (SD) & $48.0(10.2)$ & $50.1(9.1)$ \\
\hline Range & $23-73$ & $28-70$ \\
\hline \multicolumn{3}{|l|}{ Ethnicity (n (\%)) } \\
\hline White English & $68(85 \%)$ & $69(92 \%)$ \\
\hline White British & $7(9 \%)$ & $5(7 \%)$ \\
\hline Other & $5(6 \%)$ & $1(1 \%)$ \\
\hline Not stated & 4 & 5 \\
\hline \multicolumn{3}{|l|}{ Self-reported disease type (n (\%)) } \\
\hline Benign & $4(5 \%)$ & $2(3 \%)$ \\
\hline Relapsing-remitting & $35(43 \%)$ & $40(51 \%)$ \\
\hline Secondary progressive & $16(20 \%)$ & $23(29 \%)$ \\
\hline Primary progressive & $5(6 \%)$ & $8(10 \%)$ \\
\hline 'Don't know' & $21(26 \%)$ & $5(6 \%)$ \\
\hline Not stated & 3 & 2 \\
\hline \multicolumn{3}{|c|}{ APDDS score (Adapted Patient Determined Disease Steps) (n (\%)) } \\
\hline 3 or less (no limitations on walking) & $18(22 \%)$ & $15(19 \%)$ \\
\hline 4 or 5 (MS interferes with walking) & $37(46 \%)$ & $42(54 \%)$ \\
\hline $\begin{array}{l}6 \text { or more (At min., needs stick/crutch to walk } \\
100 \mathrm{~m} \text { ) }\end{array}$ & $26(32 \%)$ & $21(27 \%)$ \\
\hline Not stated & 3 & 2 \\
\hline \multicolumn{3}{|l|}{ Level of education (n (\%)) } \\
\hline \multicolumn{3}{|l|}{ Highest qualification achieved } \\
\hline No qualifications & $8(10 \%)$ & $8(10 \%)$ \\
\hline One or more GCSE (or equivalent) & $36(46 \%)$ & $29(38 \%)$ \\
\hline One or more $\mathrm{A}$ level (or equivalent) & $10(13 \%)$ & $12(16 \%)$ \\
\hline First degree (or equivalent) & $16(20 \%)$ & $19(25 \%)$ \\
\hline Higher degree/professional qualification & $9(11 \%)$ & $8(11 \%)$ \\
\hline Not stated & 5 & 4 \\
\hline \multicolumn{3}{|l|}{ Employment status (n (\%)) } \\
\hline In full-time employment ( $>30 \mathrm{~h}$ per week) & $15(18 \%)$ & $11(14 \%)$ \\
\hline In part-time employment ( $\leq 30 \mathrm{~h}$ per week) & $11(14 \%)$ & $13(17 \%)$ \\
\hline Self-employed & $4(5 \%)$ & $4(5 \%)$ \\
\hline $\begin{array}{l}\text { Not in paid employment (unemployed, in } \\
\text { education, retired, looking after home) }\end{array}$ & $51(63 \%)$ & $50(64 \%)$ \\
\hline Not stated & 3 & 2 \\
\hline \multicolumn{3}{|l|}{ Marital status (n (\%)) } \\
\hline Married/cohabiting & $63(78 \%)$ & $54(71 \%)$ \\
\hline Single & $5(6 \%)$ & $7(9 \%)$ \\
\hline Separated/divorced & $9(11 \%)$ & $14(18 \%)$ \\
\hline Widowed & $4(5 \%)$ & $1(1 \%)$ \\
\hline Not stated & 3 & 4 \\
\hline \multicolumn{3}{|l|}{ Years since diagnosis ( $\mathrm{n}(\%))$} \\
\hline$<1$ year & $2(3 \%)$ & $4(5 \%)$ \\
\hline $1-5$ years & $32(40 \%)$ & $21(27 \%)$ \\
\hline $6-10$ years & $13(16 \%)$ & $19(24 \%)$ \\
\hline $11-15$ years & $21(26 \%)$ & $12(15 \%)$ \\
\hline$\geq 16$ years & $12(15 \%)$ & $22(28 \%)$ \\
\hline Not stated & 4 & 2 \\
\hline
\end{tabular}

Percentages rounded to nearest integer and, thus, might not sum exactly to $100 \%$. CLP, current local practice; FACETS, Fatigue: Applying Cognitive behavioural and Energy effectiveness Techniques to lifestyle; GCSE, General Certificate of Secondary Education; MS, multiple sclerosis.

Scale, ${ }^{22}$ (4) the physical and psychological subscales of the MSIS-29, V.1 ${ }^{19}$ (5) the Medical Outcomes Short-Form Survey,
V.2 (SF-36v2), ${ }^{23}$ (6) health state values and quality adjusted life years (QALYs) derived from the EuroQoL 5-Dimensions questionnaire (EQ-5D) ${ }^{24}$ and the Short-form 6-Dimensions questionnaire (SF-6D) ${ }^{25}$ (7) self-reported health and social services resource utilisation with a 3 -month recall period (collected at follow-up 2 only) and (8) objective measures of physical activity over a $48 \mathrm{~h}$ period (weekday/weekend day) using ActivPAL accelerometers (total energy expenditure over $48 \mathrm{~h}$ period). ${ }^{26}$

Self-reported feedback was gathered from FACETS participants immediately after each session via a brief anonymised semistructured evaluation questionnaire returned in an envelope. Using 5-point scales the dimensions were programme content (1 'not very relevant', 5 'very relevant'), format (1 'did not work at all', 5 'worked well'), usefulness (1 'not at all useful', 5 'very useful'), pace ( 1 'too slow', 5 'too fast') and duration (1 'too short', 5 'too long').

\section{Sample size considerations and statistical analysis}

The sample size requirement was 146 participants with follow-up data based on having $85 \%$ power to detect a medium standardised effect size of $0.5^{27}$ for the primary outcome measures, using a two-sided 5\% significance level (see protocol for justification for this medium effect size). ${ }^{14}$ As a variety of fatigue measures have been used in other trials, we used standardised effect sizes to enable comparisons between them.

The main analysis was intention-to-treat but we also conducted a 'per protocol' analysis (excluding participants who attended fewer than four FACETS sessions). Data were analysed using IBM SPSS, V.18. Outcome measures were assumed to be interval-scaled, and the analysis initially focused on absolute change in outcomes at follow-up 1 (1 month postintervention) and at follow-up 2 (4 months postintervention), relative to baseline. Change scores were compared between the groups using the independent samples $t$ test with a two-sided 5\% significance level, and summarised using mean differences (95\% CIs). The GFS primary outcome is probably the most clinically relevant and so for this outcome we have also defined a clinically important improvement in fatigue as 0.5 and used the numbers-needed-to-treat statistic and the $\chi^{2}$ test for association to compare the percentage with a clinically important improvement between the two arms of the trial. This figure of 0.5 is equivalent to approximately $0.5 \mathrm{SDs}$ which was the effect size specified in the sample size calculation and recommended by others. ${ }^{28}$ We could not find any published, minimal clinically important differences (MCIDs) for the GFS subscale of the FAI in MS. However, an MCID of -0.6 points has been reported for the Fatigue Severity Scale (which shares eight of the nine GFS items, in addition to three additional ones) for systemic lupus erythematosus. ${ }^{29}$

As detailed in the protocol, ${ }^{14}$ additional prespecified supplementary analyses also (a) adjusted for baseline variability/baseline differences between treatment arms (baseline measurement, the baseline primary outcome measurements, gender, age, marital status, education level, type of MS, time since diagnosis and level of disability), (b) adjusted for any clustering effects arising from the group-based nature of FACETS using multilevel modelling, ${ }^{30}$ (c) assessed the impact of missing data ${ }^{31}$ using two approaches (the 'Last Observation Carried Forward' imputation method (which assumes no change in outcome when a data point is missing) and a mixed model approach for analysing repeated measurements) and (d) modelled all measurement occasions together for each outcome using repeated measures analysis of variance. To further aid interpretation, for primary outcomes we have also run multilevel models that include all 
time points, all baseline variables listed previously, study site and clustering effects.

Energy expenditures over $48 \mathrm{~h}$ were calculated based on the default values of the ActivPAL software package. Provided at least $24 \mathrm{~h}$ data were available, missing data were replaced by data from this period.

\section{Economic evaluation}

Cost-effectiveness analyses were undertaken from the perspective of the NHS and personal social service provider (Third Party Payer), assessing the mean incremental cost for delivery of the intervention against the effectiveness of the intervention. The primary economic endpoint is presented in cost-effectiveness analysis as the cost per unit change in GFS and also in the more policy relevant context of cost per additional person with a clinically significant improvement in fatigue. Secondary analysis estimates QALYs using the EQ-5D and SF-36 (SF-6D) and sets out findings against a cost-utility analysis. EQ-5D is used as the principal QALY measure, with SF-6D used for sensitivity analysis. QALYs were calculated over the 24-week period from baseline to follow-up 2 (0.46 years) using the area under the curve method. ${ }^{32}$ It is current convention to adjust between group differences in QALYs for baseline values of EQ-5D/SF-6D. ${ }^{33}$

Intervention costs for delivery of the FACETS programme are based on within-trial data including work sampling forms completed by clinical staff who delivered the programme. A mean net cost per participant is estimated, reflecting an incremental cost per participant. Costs were estimated using pounds sterling at 2010 unit costs. The analysis is short-term, within the trial follow-up period, and no discounting of future costs or effects is required. Sensitivity analyses have been undertaken to address key areas of uncertainty within the estimates of the intervention cost, and on assumptions included in cost estimates.

Healthcare service use data and unit cost data were combined to estimate mean healthcare costs over a 3-month period (the 3 months prior to follow-up 2) in each trial arm. Unit cost data associated with the use of health and social services in the 3 months prior to follow-up 2 were obtained from credible national sources (Personal Social Services Research Unit, ${ }^{34}$ NHS Reference costs, ${ }^{35}$ local NHS Trust cost data) (see online supplementary table S1). Non-parametric bootstrapping methods were used to estimate CIs around cost estimates for healthcare service use (see the trial protocol for further detail on methods). ${ }^{14}$

\section{RESULTS}

A total of 164 people were randomised (nine from primary care) (figure 1). First follow-up data are available for 146 (89\%) and second follow-up for 144 (88\%). Recruitment was 39 from the Bristol centre, 75 from the Southampton/Portsmouth Centre and 50 from the Poole centre. In all, 84 participants were randomised to FACETS and 80 to CLP. Overall, 10 FACETS programmes were run with group sizes ranging from 4 to 11 (mean $=7.4 ; \mathrm{SD}=2.1$ ). The percentage of participants attending individual sessions ranged from $70 \%$ (Session 5) to $81 \%$ (Session 1). A total of $72(86 \%)$ attended $\geq 4$ sessions and 66 (79\%) attended the final session (see online supplementary tables S2a and S2b). The reasons for non-attendance are reported in figure 1 .

At baseline, distributions of the descriptive statistics for the trial sample look mostly similar (table 1); since allocation was random any differences are due to chance. ${ }^{36} 37$ Table 2 and the online supplementary table S3 present descriptive statistics for the primary and secondary outcome measures, respectively, at baseline and follow-up.

\section{Primary outcomes}

The primary 'intention-to-treat' analyses and results for the primary outcome measures are shown in table 2 and online supplementary figure S1. There were statistically significantly greater improvements in fatigue self-efficacy in the FACETS arm at follow-up 1 and 2 compared with the CLP arm. By follow-up 2 , there were significantly greater improvements on the GFS scale in the FACETS arm compared with the CLP arm. Further, $40 \%$ of participants in the FACETS arm had a clinically important improvement on the GFS compared with 19\% in the CLP $\operatorname{arm}(\mathrm{p}=0.009)$, giving a numbers-needed-to-treat of 5 . There were no statistically significant differences between the FACETS arm and the CLP arm for the MSIS-29. The probability of getting three or more statistically significant differences out of six tests on the primary outcomes, just by chance, is only 0.002 .

Prespecified supplementary analyses showed that the results for the primary outcome measures were unaltered (ie, those that were statistically significant remained) by (a) adjusting for prespecified baseline characteristics and centre (with the exception of fatigue self-efficacy at 4 months (follow-up 2), where mean difference reduced from 6 to $4(-2,10), p=0.15)$, (b) adjusting for clustering, (c) using different strategies for dealing with missing data and (d) analysing all time points together using a repeated measures model.

Overall multilevel models incorporating clustering, baseline characteristics and repeated measures showed a significant main effect for fatigue self-efficacy (mean difference 6.2 (95\% CI 1.7 to 10.7$)$ ), with no interaction (ie, no evidence that the effect at 4 months is less than the effect at 1 month), a significant interaction for the GFS $(p=0.006)$, whereby the difference between the trial arms at 4 months is greater than at 1 month, and no significant difference for MSIS-29 total score. The per protocol results (including only those who attended $\geq 4$ FACETS sessions) for the primary outcomes were essentially the same as for the intention-to-treat analysis (ie, there was no impact on whether or not differences were statistically significant).

\section{Adverse events}

No adverse events, as defined in the protocol, were reported.

\section{Secondary outcomes}

Consistent with the results for the primary outcome measures, there were statistically significantly greater improvements in the secondary outcome measures of 'average fatigue' at follow-up 1 and 2, and 'fatigue right now' at follow-up 1 in the FACETS arm compared with the CLP arm (see online supplementary table S3). Further, there were greater improvements in the Vitality subscale of the SF-36 in the FACETS arm than the CLP arm at follow-up 2. In prespecified supplementary analyses, the differences seen at follow-up 2 were robust when analysed in a variety of ways, as outlined in the previous section. However, at follow-up 1 the mean difference (95\% CI) in 'average fatigue' was reduced from -0.71 to $-0.40(-0.94$ to 0.14$), \mathrm{p}=0.14$ and for 'fatigue right now' from -0.99 to -0.39 ( -1.19 to 0.41 ), $\mathrm{p}=0.33$ when we controlled for the prespecified baseline characteristics and centre.

There were no statistically significant differences between the FACETS and CLP arms at either follow-up on the MSIS-29 subscales, the Hospital Anxiety and Depression Scale, the SF-36 subscales (with the exception of 'Vitality') or the EQ-5D and SF-6D. There were also no statistically significant differences in activity levels (ActivPAL data) between the two arms at either follow-up. 


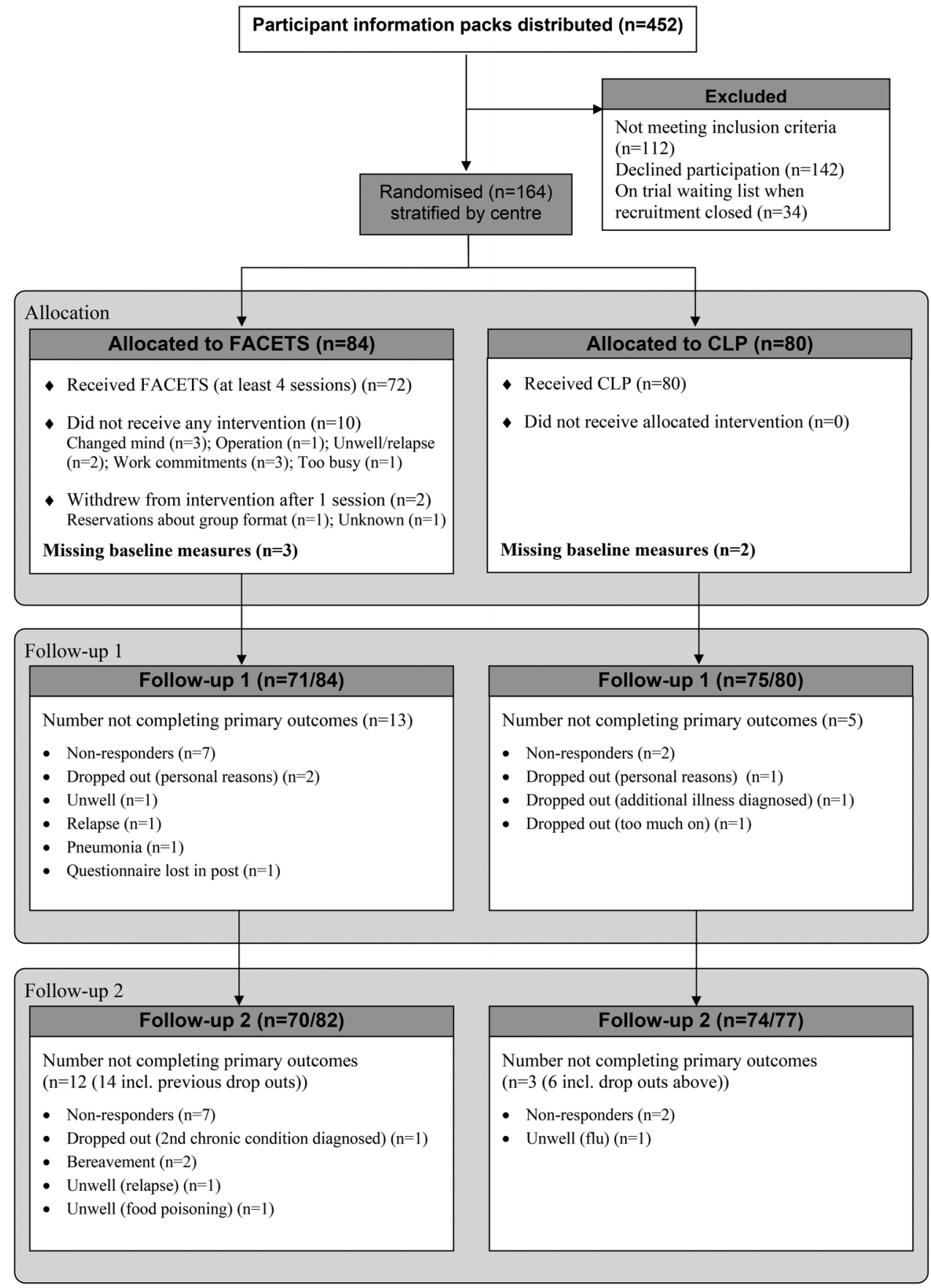

Figure 1 Flowchart showing study participation.

\section{Session evaluation questionnaires}

Out of a total of 388 session attendances, questionnaires were completed and returned for 97\%. Mean (SD) scores (on 1-5 scale, $5=$ ideal) were 4.6 (0.6) for content, 4.5 (0.7) for format, 4.6 (0.7) for usefulness and (on 1-5 scale, $3=$ ideal) $3.3(0.6)$ for pace and $3.1(0.6)$ for length (see online supplementary table S4).

\section{Economic analysis}

The estimated cost per iteration of FACETS is $£ 3625$ (see online supplementary table S5). Assuming a mean group size of eight, the mean cost per participant per FACETS programme is $£ 453$ (£518 per participant, assuming seven participants per iteration). A primary area of uncertainty in this estimate is the level of staff input time for programme delivery (mean (SD) 56.63 $(14.74) \mathrm{h}$ ). Using probabilistic sampling from this distribution, in $95 \%$ of samples the estimates of intervention cost range from $£ 331$ to $£ 585$ per participant. Assuming costs are based on input from less experienced (less-costly) health professionals (one NHS National Payscale AfC Band 7 therapist and one AfC Band 5 therapist, instead of two AfC Band 7 therapists), ${ }^{17}$ the estimated mean cost per participant is $£ 414$, with $95 \%$ of estimates in the range of $£ 311-£ 526$ per participant.

For cost-effectiveness analysis against GFS outcome, the incremental cost-effectiveness ratio was $£ 1259$ per 1-point improvement in fatigue (cost/measure of effect), or in a more policy relevant context $£ 2157$ per additional person with a clinically significant improvement in fatigue (GFS). There were no 
Table 2 Descriptive statistics and treatment effects for the primary outcome measures

\begin{tabular}{|c|c|c|c|}
\hline Outcome measure & Baseline ( $n=159)$ & Follow-up 1 (n=146) & Follow-up $2(n=144)$ \\
\hline \multicolumn{4}{|c|}{ Global Fatigue Severity subscale of the Fatigue Assessment Instrument (potential range 1-7, high scores indicate more fatigue) } \\
\hline FACETS mean (SD) & $5.60(0.98)$ & $5.48(0.92)$ & $5.26(1.03)$ \\
\hline CLP mean (SD) & $5.61(1.09)$ & $5.55(1.17)$ & $5.66(0.93)$ \\
\hline Mean diff in change from baseline $(95 \% \mathrm{Cl})^{*}$ & - & $-0.03(-0.33$ to 0.28$)$ & $-0.36(-0.63$ to -0.08$)$ \\
\hline $\mathrm{p}$ Value & - & 0.86 & 0.01 \\
\hline Std effect size & - & -0.03 & -0.35 \\
\hline \multicolumn{4}{|c|}{ Multiple Sclerosis Impact Scale-29 (potential range $0-100$, high scores indicate more impact) } \\
\hline FACETS mean (SD) & $49.6(19.1)$ & $47.3(18.2)$ & $44.9(19.2)$ \\
\hline CLP mean (SD) & $43.9(17.6)$ & $42.2(18.4)$ & $43.0(17.3)$ \\
\hline Mean diff in change from baseline $(95 \% \mathrm{Cl})^{*}$ & - & $1.44(-2.36$ to 5.24$)$ & $-1.56(-6.45$ to 3.34$)$ \\
\hline $\mathrm{p}$ Value & - & 0.46 & 0.53 \\
\hline Std effect size & - & 0.08 & -0.08 \\
\hline \multicolumn{4}{|c|}{ Fatigue Self-Efficacy Scale (potential range 10-100, high scores indicate more certainty in controlling fatigue) } \\
\hline FACETS mean (SD) & $45(17)$ & $57(17)$ & $56(19)$ \\
\hline CLP mean (SD) & $49(16)$ & $50(17)$ & $53(17)$ \\
\hline Mean diff in change from baseline $(95 \% \mathrm{Cl})^{*}$ & - & $9(4$ to 14$)$ & $6(0$ to 12$)$ \\
\hline $\mathrm{p}$ Value & - & 0.001 & 0.048 \\
\hline Std effect size & - & 0.54 & 0.36 \\
\hline
\end{tabular}

statistically significant differences between arms in EQ-5D or SF-36 (SF-6D) health state values. Observed differences in QALYs favour the CLP arm, but these are small, and not statistically significant. Thus, in the context of the cost per QALY data findings (summary statistic), CLP dominates FACETS; that is, it costs less with equal or greater benefit in QALYs. Average costs for healthcare utilisation for the FACETS arm were approximately $£ 50$ higher, although this difference was not statistically significant (see online supplementary table S6).

\section{DISCUSSION}

A novel fatigue management programme (FACETS) for people with MS is effective in reducing fatigue severity and increasing fatigue self-efficacy. Standardised effect sizes were small-tomedium. Improvements in fatigue severity were not apparent until follow-up 2 (4 months post intervention); changes in attitudes and lifestyle central to the programme are likely to take time to incorporate into daily routines. It may take longer still for these changes to impact on QOL and longer term follow-up would be required to explore this further. The ActivPAL data (see online supplementary table S3) suggest that reduced fatigue severity in the FACETS arm was not primarily achieved via diminished activity levels. Improvements in fatigue self-efficacy, while significant at first follow-up, were only marginally so at follow-up 2, and did not remain significant after supplementary analyses adjusting for prespecified baseline variables and centre. Relative to the CLP arm, there were no changes in diseasespecific QOL. Ratings on evaluation questionnaires indicated high overall satisfaction, and combined with high attendance rates, suggested the programme was well received.

The estimated additional cost for FACETS is modest at $£ 453$, and it has shown an important difference in the proportion of patients with a clinically significant improvement in fatigue (GFS) as well as improvements on the Vitality subscale of the SF-36 and in fatigue self-efficacy. However, the costeffectiveness case is equivocal as it is difficult to interpret these benefits in the context of overall health related QOL, given the absence of evidence in the trial to support QALY gains. Given the paucity of treatment options for fatigue in MS, the FACETS intervention may present as a value for money intervention. We suggest that decision-makers may be persuaded by the potential for FACETS to be cost-effective as at the estimated cost for FACETS it would only take a small incremental change in QALYs (a change of 0.015 QALYs) to indicate the intervention was cost-effective at a cost per QALY threshold commonly applied in the UK National Institute for Health and Clinical Excellence setting. The evidence from the current trial is unclear, and we discuss this further below.

The only other RCT to explore the effectiveness of a cognitive behavioural approach for the management of MS-fatigue took place in New Zealand. ${ }^{11}$ This trial differed from ours in that it involved one-to-one cognitive behavioural therapy delivered by one clinical psychologist in a single centre to relatively small numbers of people with MS and had an active comparator (relaxation) and used a different measure of fatigue. This makes direct comparison of the results problematic. In a health service like the UK NHS where psychologists working with people with MS are scarce, this approach may prove impractical.

\section{Strengths and limitations}

This is the largest RCT to explore a cognitive behavioural approach to fatigue management for people with MS. Trial quality was high in that treatment allocation was concealed, the recruitment target met and sample attrition low. A key strength of this trial is its pragmatic nature and high external validity: the programme was compared against what is currently happening in the NHS, in a variety of locations, and was mostly delivered by individuals not involved in developing the programme but practising clinically. It thus offers a test of the intervention in an environment very close to real clinical practice. Effect sizes were modest but pragmatic trials generally yield smaller effect sizes than tightly controlled RCTs. Given the high prevalence of fatigue in MS and its debilitating impact, small/medium improvements may be important. 
We were not able to assess the representativeness of the sample by comparing trial participant characteristics with those who were not recruited as these data were not collected for the latter group. It is possible that there might have been a recruitment bias towards individuals who were more amenable to a non-pharmacological approach.

A potential criticism of pragmatic trials such as ours is that the FACETS arm received more health professional contact, time and attention than the CLP arm and it might be this, rather than programme content, which produced improvements. However, despite considering outcomes covering a range of dimensions, only those specifically related to the purpose of the FACETS programme (ie, fatigue and fatigue self-efficacy) were statistically significant. If there was an impact of attention, one could hypothesise the observed differences would be less focused.

In keeping with the pragmatic nature of this trial, to increase external validity no attempt was made to restrict or control trial participants' access to current local practice or to standardise it across healthcare settings or treatment arms. In none of the centres was best practice to do nothing; participants will have received a variety of advice depending on local service/individual need. Collecting accurate detailed information at an individual level on the type/quantity of advice received as part of current local practice was outside the scope of the trial and is therefore a limitation.

We did not formally assess treatment fidelity. Measuring fidelity would have required either having an observer or audio-/ video-recording sessions and these approaches can impact on participants' willingness to participate in research and on the dynamics of group sessions. FACETS is manualised with a detailed description of content to be delivered, a suggested script for facilitators, guidance notes and a detailed checklist of key aspects to be covered. Observations of sessions, feedback from the facilitators during the three centre pilot study, facilitators' debriefs and evaluation of participant feedback on each session suggested that the intervention was being delivered as intended.

We acknowledge that specifying three primary outcomes and two follow-up points (ie, six tests) inflates the risk of a Type I error. However, the probability of obtaining statistically significant differences on $3 / 6$ tests (independent) by chance is very small $(\mathrm{p}=0.002)$ and supplementary analysis using repeated measures analysis of variance to analyse all time points together did not affect the findings. The follow-up period in this trial was relatively short (4 months). Longer follow-up periods would be needed to assess the longevity of the results. The FACETS programme encourages changes in behaviour and thinking which, if maintained, have the potential for longer term benefit.

In the UK NHS and elsewhere, cost per QALY is a common basis for decision-making related to funding healthcare interventions. $^{38-40}$ The QALY data here indicate no statistically significant difference between the trial arms, and a trend towards CLP dominating FACETS (ie, the latter costs more and has equal or slightly less benefit in QALYs). However, there is some basis for being cautious about these findings, and for considering the potential for FACETS to offer a cost-effectiveness profile that may be attractive to decision-makers, as there is a difference in effectiveness for fatigue, a key aspect of patient reported outcome, even though it is not reflected in the QALY outcomes. There is a growing evidence base to suggest that the EQ-5D and SF-36 generic measures may lack both relevance and sensitivity to changes in health related QOL in MS. These measures, although widely used, may not address important areas of impact specific to MS, most notably fatigue, ${ }^{41-44}$ and both have been found to lack responsiveness. ${ }^{45}$ The relatively small sample size in the current study could have contributed to the insensitivity of QALY measures to differences in fatigue. Recent research on condition-specific preference-based measures for MS, using a large observational study, has indicated that where there is an improvement in MSIS-29 subscale scores (as seen in the current study although not statistically significant), there is likely to be a QALY gain. ${ }^{42}$ Therefore, it may be that FACETS does have the potential to demonstrate QALY gains, although the current study has not presented evidence of this using the generic health status measures.

This intervention could be a clinically useful, relatively inexpensive addition to current local practice in appropriate hospital/community settings. It could be readily incorporated into existing services, facilitating the integration of psychology-based approaches into patient care.

Correction notice This paper has been amended since it was published Online First. The legend for figure 1 has been incorrectly titled. Originally it was written as follows: "Mean differences and 95\% Cls on the primary outcomes at baseline, follow-up 1 (1 month postintervention) and follow-up 2 (4 months postintervention). CLP, current local practice; FACETS, Fatigue: Applying Cognitive behavioural and Energy effectiveness Techniques to lifeStyle." It has now been corrected to the following legend: "Flowchart showing study participation."

Acknowledgements Thanks to all the participants who took part and to the clinicians who identified the potential participants for the trial. Thanks to Ms Felicity Burgess for supporting recruitment at the Southampton centre. Thanks to Dr Sara Demain, Mrs Caroline Birch, Ms Charlie Ewer-Smith, Mrs Jo Kileff, Mrs Jenn Gash and Mrs Sheila Chartres for delivering the FACETS Programme. Thanks to Dr Reuben Ogollah for data extraction from the ActivPALs ${ }^{\mathrm{TM}}$. Thanks to Mr Geoff Linder and Mr Tim Worner for patient and public involvement. Thanks to Mrs Chris Richards, Mrs Moira Hawey and Mrs Louise Ward for data entry. Thanks for support from the Comprehensive Clinical Research Network and the Primary Care Network. Thanks to Ms Sally Johnson for contributing to facilitation of the FACETS programme during the pilot work.

Contributors ST: conception, design, acquisition of data, analysis, interpretation, drafted article. PT: chief investigator, conception, design, analysis, interpretation, drafted article. PK and RJ: design, acquisition of data, interpretation, critically reviewed article. CG: design, analysis, interpretation, drafted economic aspects, critically reviewed article. AN and VS: design, delivered fatigue management programme, acquisition of data, interpretation, critically reviewed article. ADS: design, delivered fatigue management programme, critically reviewed article. RB and KTG: design, interpretation, critically reviewed article. $\mathrm{CH}$ : design, clinical overview of trial, interpretation, critically reviewed article.

Funding This research was funded by the Multiple Sclerosis Society of Great Britain and Northern Ireland (Grant number 846/06).

Competing interests All authors have completed the Unified Competing Interests form at http://www.icmje.org/coi_disclosure.pdf (available on request from the corresponding author) and declare: all authors had financial support from the Multiple Sclerosis Society of Great Britain and Northern Ireland for the submitted work; no financial relationships with any organisations that might have an interest in the submitted work in the previous 3 years. RB is the chair of the MS Society Grant Review Panel for Care and Services Research. PT and CG are members of the MS Society Grant Review Panel for Care and Services Research. PT is a member of the Advisory Board for the Sativex Registry. The Board provides an independent review of safety data for patients prescribed Sativex. Bournemouth University receives a fee from GW Pharma to cover time spent at meetings, and travel expenses.

\section{Patient consent Obtained.}

Ethics approval South West-Central Bristol Research Ethics Committee (ref: 08/H0106/2).

Provenance and peer review Not commissioned; externally peer reviewed.

Open Access This is an Open Access article distributed in accordance with the Creative Commons Attribution Non Commercial (CC BY-NC 3.0) license, which permits others to distribute, remix, adapt, build upon this work non-commercially, and license their derivative works on different terms, provided the original work is properly cited and the use is non-commercial. See: http://creativecommons.org/ licenses/by-nc/3.0/

\section{REFERENCES}

1 Krupp LB, Serafin DJ, Christodoulouu C. Multiple sclerosis-associated fatigue. Expert Rev Neurother 2010;10:1437-47. 
2 Branas P, Jordan RE, Fry-Smith A, et al. Treatments for fatigue in multiple sclerosis: a rapid and systematic review. Health Technol Ass 2000;4:1-61.

3 Costello K, Harris C. Differential diagnosis and management of fatigue in multiple sclerosis: considerations for the nurse. J Neurosci Nurs 2003;35:139-48.

4 Grima DT, Torrance GW, Francis G, et al. Cost and health related quality of life consequences of multiple sclerosis. Mult Scler 2000;6:91-8.

5 White $C P$, White $M B$, Russell CS. Invisible and visible symptoms of multiple sclerosis: which are more predictive of health distress? J Neurosci Nurs 2008;40:85-95.

6 Bakshi R. Fatigue associated with multiple sclerosis: diagnosis, impact and management. Mult Scler 2003;9:219-27.

7 Mathiowetz VG, Matuska KM, Finlayson ML, et al. One-year follow-up to a randomized controlled trial of an energy conservation course for persons with multiple sclerosis. Int J Rehabil Res 2007;30:305-13.

8 Blikman $\mathrm{L}$, Huisstede BM, Koojimans $\mathrm{H}$, et al. Effectiveness of energy conservation treatment in reducing fatigue in multiple sclerosis: a systematic review and metaanalysis. Arch Phys Med Rehabil Published Online First: 11 Feb 2013. doi:10.1016/ j.apmr.2013.01.025

9 Lee $D$, Newell $R$, Ziegler $L$, et al. Treatment of fatigue in multiple sclerosis: a systematic review of the literature. Int I Nurs Pract 2008;14:81-93.

10 van Kessel K, Moss-Morris R: Understanding multiple sclerosis fatigue: a synthesis of biological and psychological factors. J Psychosom Res 2006;61:583-5.

11 van Kessel K, Moss-Morris R, Willoughby E, et al. A Randomized controlled trial of cognitive behavior therapy for multiple sclerosis fatigue. Psychosom Med 2008;70:205-13

12 Thomas PW, Thomas S, Hillier C, et al. Psychological interventions for multiple sclerosis. Cochrane Database Syst Rev 2006;1:CD004431. doi: 10.1002/14651858. CD004431.pub2

13 Thomas S, Thomas PW, Nock A, et al. Development and preliminary evaluation of a cognitive behavioural approach to fatigue management in people with multiple sclerosis. Patient Educ Couns 2010:78:204-10.

14 Thomas PW, Thomas S, Kersten P, et al. Multi-centre parallel arm randomised controlled trial to assess the effectiveness and cost-effectiveness of a group-based cognitive behavioural approach to managing fatigue in people with multiple sclerosis BMC Neurol 2010:10:43.

15 Boutron I, Moher D, Altman DG, et al.; for the CONSORT group. Extending the CONSORT Statement to randomized trials of nonpharmacologic treatment: explanation and elaboration. Ann Intern Med 2008;148:295-309.

16 Krupp LB, LaRocca NG, Muir-Nash J, et al. The fatigue severity scale. Application to patients with multiple sclerosis and systemic lupus erythematosus. Arch Neurol 1989;10:1121-3.

17 NHS Careers. Pay and benefits for AHP staff. http://www.nhscareers.nhs.uk/exploreby-career/allied-health-professions/pay-and-benefits-for-ahp-staff/ (accessed 30 Dec 2012).

18 Schwartz JE, Jandorf L, Krupp L. The measurement of fatigue: a new instrument. J Psychosom Res 1993:37:753-62.

19 Hobart JC, Riazi A, Lamping D, et al. Improving the evaluation of therapeutic interventions in multiple sclerosis: development of a patient-based measure of outcome. Health Technol Assess 2004;8:1-60.

20 Schwartz CE, Coulthard-Morris L, Zeng Q, et al. Measuring self-efficacy in people with multiple sclerosis: a validation study. Arch Phys Med Rehabil 1996;77:394-8.

21 Hann D, Jacobsen $P$, Azzarello $L$, et al. Measurement of fatigue in cancer patients: Development and validation of the Fatigue Symptom Inventory. Qual Life Res 1998; 7:301-10.

22 Zigmond AS, Snaith RP: The Hospital Anxiety and Depression Scale. Acta Neurol Scand 1983;67:361-70.
23 Ware JE, Sherbourne CD. The MOS 36-item Short-Form health survey (SF-36):I. Conceptual framework and item selection. Med Care 1992;30:473-83.

24 Dolan P. Modeling Valuations for EuroQol Health States. Med Care 1997;35:1095-108

25 Brazier J, Roberts J, Deverill M. The estimation of a preference-based measure of health from the SF-36. J Health Econ 2002;21:271-92.

26 Ryan CG, Grant PM, Tigbe WW, et al. The validity and reliability of a novel activity monitor as a measure of walking. Br J Sports Med 2006;40:779-84.

27 Cohen J. Statistical power analysis for the behavioral sciences. 2nd edn. Hillsdale: NJ Lawrence Erlbaum Associates, 1988.

28 Norman GR, Sloan JA, Wyrwich KW. Interpretation of changes in health-related quality of life. The remarkable universality of half a standard deviation. Med Care 2003;41:582-92.

29 Goligher EC, Pouchot J, Brant R, et al. Minimal clinically important difference for 7 measures of fatigue in patients with systemic lupus erythematosus. J Rheumatol 2008;35:635-42.

30 Bauer DJ, Sterba SK, Hallford DD. Evaluating group based interventions when control participants are ungrouped. Multivar Behav Res 2008;43:210-36.

31 Lane P. Handling drop-out in longitudinal clinical trials: a comparison of the LOCF and MMRM approaches. Pharm Stat 2008;7:93-106.

32 Brazier JE, Ratcliffe J, Salomon A, et al. Measuring and valuing health for economic evaluation. Oxford: Oxford University Press, 2007.

33 Manca A, Hawkins N, Sculpher M. Estimating mean QALYs in trial-based cost effectiveness analysis: the importance of controlling for baseline utility. Health Econ 2005:4:87-496.

34 Curtis L. Unit costs of health and social care. Personal social services research unit (PSSRU), Canterbury. UK: University of Kent, 2010.

35 Department of Health. NHS Reference Costs 2009-2010, 2011. http://www.dh.gov. uk/en/Publicationsandstatistics/Publications/PublicationsPolicyAndGuidance/ DH_123459 (accessed 30 Dec 2012).

36 CONSORT Statement website. http://www.consort-statement.org/consort-statement/ 13-19--results/item 15 baseline-data/ (accessed 7 Mar 2013).

37 Altman DG, Doré CJ. Randomisation and baseline comparisons in clinical trials. Lancet 1990:335:149-53.

38 National Institute for Health and Clinical Excellence (NICE). Guide to the methods of technology appraisal. London, UK: NICE, June 2008.

39 Australian Government Department of Health and Ageing. Pharmaceutical Benefits Advisory Committee. Guidelines for preparing submissions to the Pharmaceutical Benefits Advisory Committee (Version 4.3). Barton, Australia: Australian Government. Department of Health and Ageing, December 2008.

40 Canadian Agency for Drugs and Technologies in Health. Guidelines for the economic evaluation of health technologies: Canada, 3rd edn. Ottawa: Canadian Agency for Drugs and Technologies in Health, 2006.

41 Hemmett L, Holmes J, Barnes $M$, et al. What drives quality of life in multiple sclerosis? Q J Med 2004:97:671-6.

42 Hobart J, Freeman J, Lamping D, et al. The SF-36 in multiple sclerosis: why assumptions must be tested. J Neurol Neurosurg Psychiatry 2001;71:363-93.

43 Opara JA, Jaracz K, Brola W. Quality of life in multiple sclerosis. J Med Life 2010;3:352-8.

44 Fisk JD, Brown MG, Sketris IS, et al. A comparison of health utility measures for the evaluation of multiple sclerosis treatments. I Neurol Neurosurg Psychiatry 2005;76:58-63.

45 Hawton A, Green C, Telford C, et al. Using the Multiple Sclerosis Impact Scale in treatment decision-making: Mapping from the MSIS-29 version 2 to the EQ-5D and the SF-6D. Value Health 2012;15:1084-91. 